\title{
Stochastic Reliability Analysis for Drum System of Hang Type Centrifuge
}

\author{
Xujuan Yang ${ }^{1, a^{*}}$, Yulong $\mathrm{Li}^{1, \mathrm{~b}}$, Zhaojun $\mathrm{Li}^{1, \mathrm{c}}$, \\ Rui Yan ${ }^{1, d}$, Zhen Zhang ${ }^{1, e}$ \\ ${ }^{1}$ Guangxi Key Laboratory of Manufacturing System \& Advanced Manufacturing Technology, \\ College of Mechanical Engineering, Guangxi University, Nanning 530004, China \\ ayxj413@163.com, b13211327285@163.com, glenlzj@126.com, \\ d2391021864@qq.com, e879822534@qq.com
}

Keywords: drum, stochastic reliability, invalid.

Abstract. A stochastic reliability model of the drum system of a hang type centrifuge drum is presented using stochastic reliability theory, where the stochastic reliability index and invalid probability of the drum system are determined by checking point algorithm and the system functional functions are built according to the criterion that absolute value difference between natural frequencies and excitation frequencies should be less than specific values. An actual case is calculated and analyzed and the correctness of the model is verified by Monte Carlo method, the speed range of the drum system is pointed out where failure may occur easily and the low reliability zone is obtained which should be avoided in actual operation.

\section{Introduction}

The hang type centrifuge is an intermittent centrifuge and is an important equipment in the process of separating sugar. Its working principle is using the centrifugal force to separate the white sugar from the massecuite mother liquor ${ }^{[1]}$. The separation ability of the hang type centrifuge is improved with the increase of its speed. Many companies often use the method of increasing centrifuge speed to improve its work efficiency. But with the increase of the centrifuge speed, the vibration problem of the centrifuge is becoming more and more serious, which may cause unnecessary casualties and property loss ${ }^{[2]}$. Thus, it is necessary to carry out the reliability analysis of the hang type centrifuge. At present, the reliability problems of centrifuge have been extensively studied. For example, the reliability of the rotor, motor and frequency converter of the ultracentrifuge were studied by using fault tree analysis method by $\mathrm{Lu} \mathrm{Hua}^{[3]}$. The reliability of the spindle and drum of a reprocessing centrifuge was analyzed by the fault tree analysis method by Zhou Qiwang ${ }^{[4]}$, and he also gave us some methods which could improve the reliability of the reprocessing centrifuge components. The high-peed vertical tumbler centrifuge spindle reliability design were studied by using fuzzy reliability theory by Ren Yundan ${ }^{[5]}$, and the fuzzy comprehensive evaluation of the spindle reliability was carried out. The hang type centrifuge is the key equipment in the sugar enterprises, and the drum is the main stressed body. The drum tends to cause resonance failure under the action of forced vibration. However, the reliability study about the hang type centrifuge drum has been rarely reported. Therefore, the reliability of the drum needs to be studied. There are many random parameters in the drum system, which leads to the result that the natural frequencies and the excitation frequencies of the system are discrete. So it is necessary to build the reliability model of the drum system.

Based on the dynamic model of the drum system, according to the criterion that absolute value difference between natural frequencies and excitation frequencies should be less than specific values, the reliability of the drum system under forced vibration failure mode is studied by stochastic reliability theory. 


\section{Dynamic model}

The shell deformation theory is used to analyze the drum system of a hang type centrifuge. Compared to the diameter of the centrifuge drum, the thickness of that is much smaller, so the drum wall is viewed as a shell in the paper and triangle plate element is used to build the drum wall. With consideration of fluid-structure interaction between the drum and massecuite, the dynamic model by using finite element method can be expressed as ${ }^{[6]}$ :

$$
\left(\boldsymbol{M}+\boldsymbol{M}_{a}\right) \ddot{\boldsymbol{U}}+\boldsymbol{C} \dot{\boldsymbol{U}}+\boldsymbol{K} \boldsymbol{U}=\boldsymbol{F}
$$

Where $\boldsymbol{U}$ is the displacement vector of the drum system, $\boldsymbol{M}$ is the mass matrix of the drum, $\boldsymbol{M}_{a}$ is the additional mass matrix caused by the fluid element, $\boldsymbol{K}$ is stiffness matrix of the drum system, $\boldsymbol{C}$ is the damp matrix of the drum system, and $\boldsymbol{F}$ is the generalized force column vector.

\section{Stochastic reliability mode}

\section{Design principle of stochastic reliability}

Randomness is a kind of uncertainty due to the uncertainty of the causal relationship, the reliability analysis model considering the uncertainty is called stochastic reliability model. There are obvious uncertain factors in the system of hang type centrifuge drum, such as the density of massecuite and speed. So it's necessary to establish a stochastic reliability model.

The working state of the drum system is expressed by functional functions as follows:

$$
\boldsymbol{Z}=g(\boldsymbol{x})=g\left(x_{1}, x_{2}, \ldots, x_{n}\right)
$$

Where $\boldsymbol{x}=\left(x_{1}, x_{2}, \ldots, x_{n}\right)^{T}$ is a set of uncertain parameters of the drum system.

The stochastic reliability of the drum system can be expressed as:

$$
R=P(Z>0)
$$

\section{Stochastic reliability under forced vibration failure mode}

The failure probability is the probability that the product can not be completed within specified time and conditions. There are obvious random parameters in the drum system, thus the natural frequencies and excitation frequencies of the drum are discrete. When analyzing the vibration of the drum system, the system functional functions considering the criterion that absolute value difference between natural frequencies and excitation frequencies should be less than specific values are built as follows:

$$
g\left(\omega_{m}, p_{n}\right)=\left|\omega_{m}-p_{n}\right|-c
$$

Where $c$ is an empirical parameter, $\omega_{m}$ is the $m$-th natural frequency of the system and $p_{n}$ is the $n$-th excitation frequency of the system.

In order to make the calculation simple, the functional functions of the system are expressed as

$$
g\left(\omega_{m}, p_{n}\right)= \begin{cases}g_{1}=\omega_{m}-p_{n}-c & p_{n} \leq \omega_{m} \\ g_{2}=p_{n}-\omega_{m}-c & p_{n}>\omega_{m}\end{cases}
$$

The system functional functions are equivalent to the parallel combination of the two simple sub functions. The failure probabilities of $g_{1}$ and $g_{2}$ are expressed by $P_{1}$ and $P_{2}$, and then the failure probability function of the system under forced vibration failure mode can be expressed as

$$
\begin{aligned}
P & =P(g \leq 0)=P\left(g_{1} \leq 0 \bigcap g_{2} \leq 0\right) \\
& =P\left(g_{1} \leq 0\right)+P\left(g_{2} \leq 0\right)-P\left(g_{1} \leq 0 \bigcup g_{2} \leq 0\right) \\
& =P_{1}+P_{2}-1
\end{aligned}
$$


In order to facilitate the derivation, the frequency factor of the system with uncertainty parameters is expressed as

$$
p(\boldsymbol{x})=\sum_{i=1}^{n} a_{i} \omega_{i}(\boldsymbol{x})+\Omega(\boldsymbol{x})
$$

Where $\omega_{i}(\boldsymbol{x})$ is the $i$-th natural frequency of the system, $\Omega(\boldsymbol{x})$ is the excitation frequency of the drum system, $\omega_{i}(\boldsymbol{x})$ and $\Omega(\boldsymbol{x})$ are related to the uncertainty variable $\boldsymbol{x}, n$ is the number of the free degree of the system and $a_{i}$ is the coefficient of the natural frequency of the system.

Substituting equation (7) to equation (5), the functional functions of the system can be expressed as

$$
g\left(\omega_{m}, p_{n}\right)=\left\{\begin{array}{l}
g_{1}(\boldsymbol{x})=\left(1-a_{m}\right) \omega_{m}(\boldsymbol{x})-\sum_{i=1, i \neq m}^{n} a_{i} \omega_{i}(\boldsymbol{x})-\Omega(\boldsymbol{x})-c \\
g_{2}(\boldsymbol{x})=\left(a_{m}-1\right) \omega_{m}(\boldsymbol{x})+\sum_{i=1, i \neq m}^{n_{\infty}} a_{i} \omega_{i}(\boldsymbol{x})+\Omega(\boldsymbol{x})-c
\end{array}\right.
$$

The functional functions are carried out at the point $\boldsymbol{x}_{1}^{*}=\left[x_{11}^{*}, x_{12}^{*}, \cdots, x_{1 n}^{*}\right]^{T}$ and $\boldsymbol{x}_{2}^{*}=\left[x_{21}^{*}, x_{22}^{*}, \cdots, x_{2 n}^{*}\right]^{T}$ with Taylor expansion by using checking point algorithm. The nonlinear parts of the expansion of the above are ignored, then the reliability index and failure probability of $g_{1}$ and $g_{2}$ can be expressed as

$$
\begin{aligned}
& \left\{\begin{array}{c}
\beta_{1}=\frac{\left.\sum_{t=1}^{n}\left(\frac{\partial g_{1}(\boldsymbol{x})}{\partial x_{t}}\right)\right|_{x=x_{1}^{*}} \cdot\left(\mu_{x_{t}}-x_{1 t}^{*}\right)}{\left[\left.\sum_{t=1}^{n}\left(\frac{\partial g_{1}(\boldsymbol{x})}{\partial x_{t}}\right)^{2}\right|_{x=x_{1}^{*}} \cdot \sigma_{x_{t}}^{2}\right]^{\frac{1}{2}}} \\
\beta_{2}=\frac{\left.\sum_{t=1}^{n}\left(\frac{\partial g_{2}(\boldsymbol{x})}{\partial x_{t}}\right)\right|_{x=x_{1}^{*}} \cdot\left(\mu_{x_{t}}-x_{2 t}^{*}\right)}{\left[\left.\sum_{t=1}^{n}\left(\frac{\partial g_{2}(\boldsymbol{x})}{\partial x_{t}}\right)^{2}\right|_{x=x_{2}^{*}} \cdot \sigma_{x_{t}}^{2}\right]^{\frac{1}{2}}}
\end{array}\right. \\
& \left\{\begin{array}{l}
P_{1}=\Phi\left(-\beta_{1}\right) \\
P_{2}=\Phi\left(-\beta_{2}\right)
\end{array}\right.
\end{aligned}
$$

Where

$$
\left\{\begin{array}{l}
\left.\frac{\partial g_{1}(\boldsymbol{x})}{\partial x_{t}}\right|_{x=x_{1}^{*}}=\left.\left(1-a_{m}\right) \cdot\left(\frac{\partial \omega_{m}(\boldsymbol{x})}{\partial x_{t}}\right)\right|_{x=x_{1}^{*}}-\left.\sum_{i=1,1 ; m}^{n} a_{i} \cdot\left(\frac{\partial \omega_{i}(\boldsymbol{x})}{\partial x_{t}}\right)\right|_{x=x_{1}^{*}}-\left.\frac{\partial \Omega(\boldsymbol{x})}{\partial x_{t}}\right|_{x=x_{1}^{*}} \\
\left.\frac{\partial g_{2}(\boldsymbol{x})}{\partial x_{t}}\right|_{x=x_{2}^{*}}=\left.\left(a_{m}-1\right) \cdot\left(\frac{\partial \omega_{m}(\boldsymbol{x})}{\partial x_{t}}\right)\right|_{x=x_{2}^{*}}+\left.\sum_{i=1, i, m}^{n} a_{i} \cdot\left(\frac{\partial \omega_{i}(\boldsymbol{x})}{\partial x_{t}}\right)\right|_{x=x_{2}^{*}}+\left.\frac{\partial \Omega(\boldsymbol{x})}{\partial x_{t}}\right|_{x=x_{2}^{*}}
\end{array}\right.
$$

Generally speaking, the value of the $\partial \Omega(\boldsymbol{x}) / \partial x_{t}$ is known, and the values of $\partial \omega_{i}(\boldsymbol{x}) / \partial x_{t}$ and $\partial \omega_{m}(\boldsymbol{x}) / \partial x_{t}$ can be obtained as follows: 


$$
\left\{\begin{array}{l}
\frac{\partial \omega_{i}(\boldsymbol{x})}{\partial x_{t}}=\frac{1}{2 \omega_{i}(\boldsymbol{x})}\left(\eta_{i}^{\mathrm{T}}(\boldsymbol{x})\left[\begin{array}{l}
\frac{\partial \boldsymbol{K}(\boldsymbol{x})}{\partial x_{t}}-\omega_{i}^{2}(\boldsymbol{x}) \frac{\partial \boldsymbol{M}(\boldsymbol{x})}{\partial x_{t}} \\
-\omega_{i}^{2}(\boldsymbol{x}) \frac{\partial \boldsymbol{M}(\boldsymbol{x})}{\partial x_{t}}
\end{array}\right] \boldsymbol{\eta}_{i}(\boldsymbol{x})\right) \\
\frac{\partial \omega_{p}(\boldsymbol{x})}{\partial x_{t}}=\frac{1}{2 \omega_{p}(\boldsymbol{x})}\left(\eta_{p}^{\mathrm{T}}(\boldsymbol{x})\left[\begin{array}{l}
\frac{\partial \boldsymbol{K}(\boldsymbol{x})}{\partial x_{t}}-\omega_{p}^{2}(\boldsymbol{x}) \frac{\partial \boldsymbol{M}(\boldsymbol{x})}{\partial x_{t}} \\
-\omega_{p}^{2}(\boldsymbol{x}) \frac{\partial \boldsymbol{M}(\boldsymbol{x})}{\partial x_{t}}
\end{array}\right] \boldsymbol{\eta}_{p}(\boldsymbol{x})\right.
\end{array}\right]
$$

Where $\boldsymbol{\eta}_{i}$ is the $i$-th modal coordinate of the drum system, $\boldsymbol{\eta}_{p}$ is the $p$-th modal coordinate of the drum system.

Above all, the failure probability of the drum system can be expressed as

$$
P=\Phi\left(-\beta_{1}\right)+\Phi\left(-\beta_{2}\right)-1
$$

Then the reliability of the drum system under forced vibration failure mode can be expressed as

$$
R=1-P
$$

\section{Case simulation}

Taking a drum of a hang type centrifuge for example, the working speed of the hang type centrifuge is $1060 \mathrm{r} / \mathrm{min}$, the maximum speed is $1300 \mathrm{r} / \mathrm{min}$, the diameter of the drum is $1600 \mathrm{~mm}$, the height of the drum is $1097 \mathrm{~mm}$, and the density of the drum material is $7850 \mathrm{~kg} / \mathrm{m}^{3}$. Taking the density of massecuite as random parameter to study the reliability of the drum system, then the random variable is $\boldsymbol{x}=[\rho]$, the initial density of the massecuite is $1500 \mathrm{~kg} / \mathrm{m}^{3}$.

\section{Stochastic reliability of the drum system}

According to calculation, the first three order natural frequencies of the drum system are shown in table 1 .

Table 1. The first three order natural frequencies of the drum system

\begin{tabular}{|l|c|c|c|}
\hline order & 1 & 2 & 3 \\
\hline natural frequency $(\mathrm{Hz})$ & 12.23 & 13.16 & 27.41 \\
\hline
\end{tabular}

The value of the empirical parameter $\mathrm{c}$ is defined as 5 percent of the first order natural frequency, i.e. $c=0.612 \mathrm{~Hz}$. The reliability of the drum system is shown as fig. 1 .

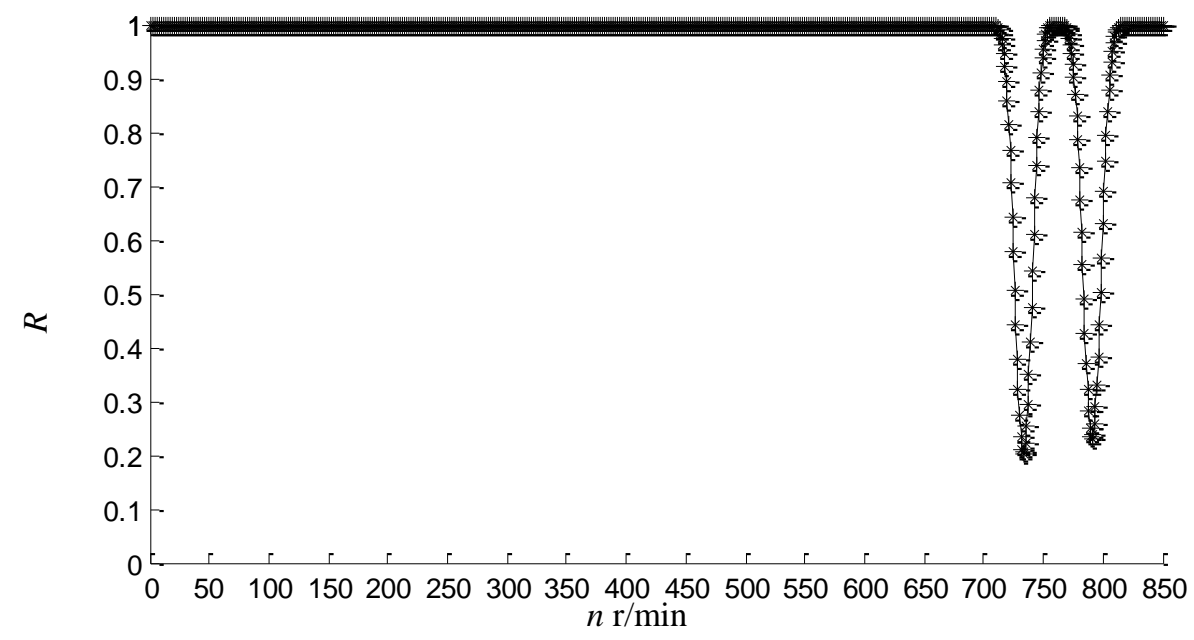

Fig. 1. Stochastic reliability of the drum system

As shown in Fig. 1, the reliability of the drum system is relatively low when the spindle speed 
varies from $720 \mathrm{r} / \mathrm{min}$ to $750 \mathrm{r} / \mathrm{min}$ or $770 \mathrm{r} / \mathrm{min}$ to $810 \mathrm{r} / \mathrm{min}$, where the resonance failure is easy to occur. Therefore, these resonance regions should be avoided.

\section{Verification of the accuracy of the system reliability model}

Monte-Carlo method is a useful method to verify the accuracy of stochastic reliability models, where repeated random sampling for simulating the probability and statistics of the research object are used to build a probability model or random process, so as to make its parameters equal to the solution of the problem ${ }^{[7,8]}$. To reduce the running time of the simulation program, the reliability of the drum is checked only during the speed of $700 \mathrm{r} / \mathrm{min}$ to $850 \mathrm{r} / \mathrm{min}$. The stochastic reliability of the system obtained by Monte-Carlo method is shown in Fig. 2, and the comparison of Fig. 1 and Fig. 2 is shown in Fig. 3.

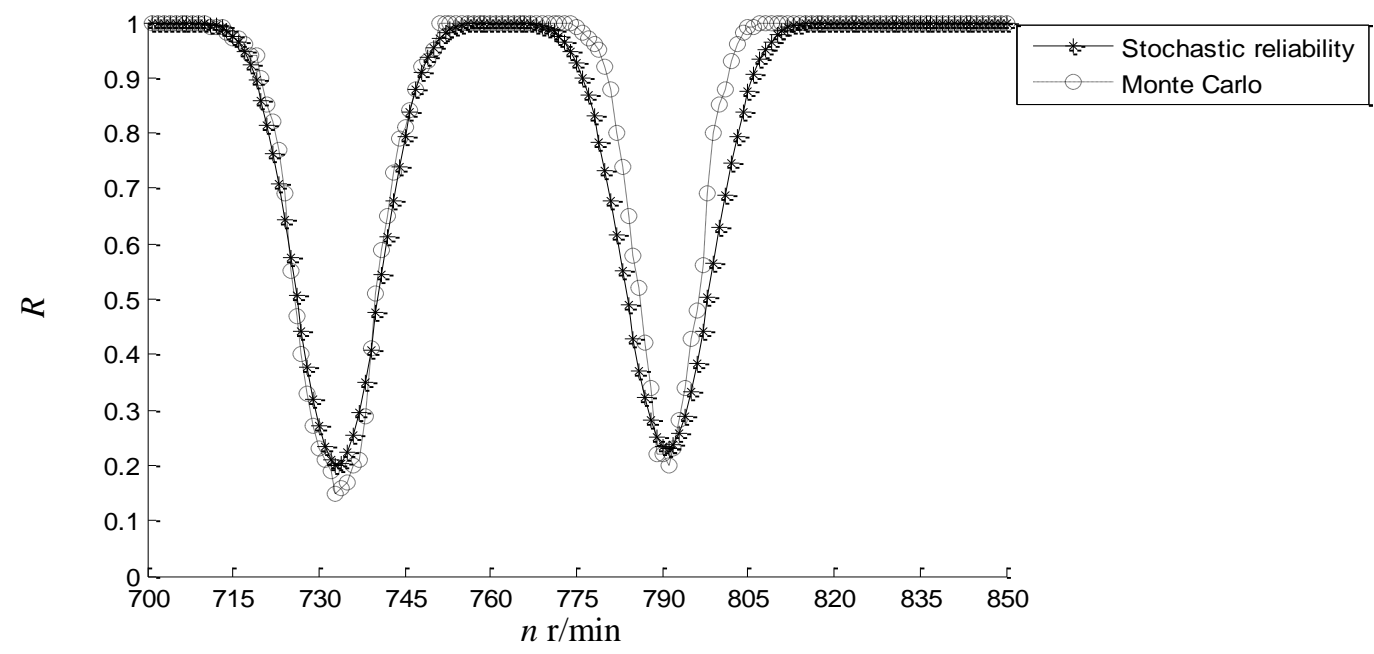

Fig. 2. Reliability of drum system by Monte-Carlo method

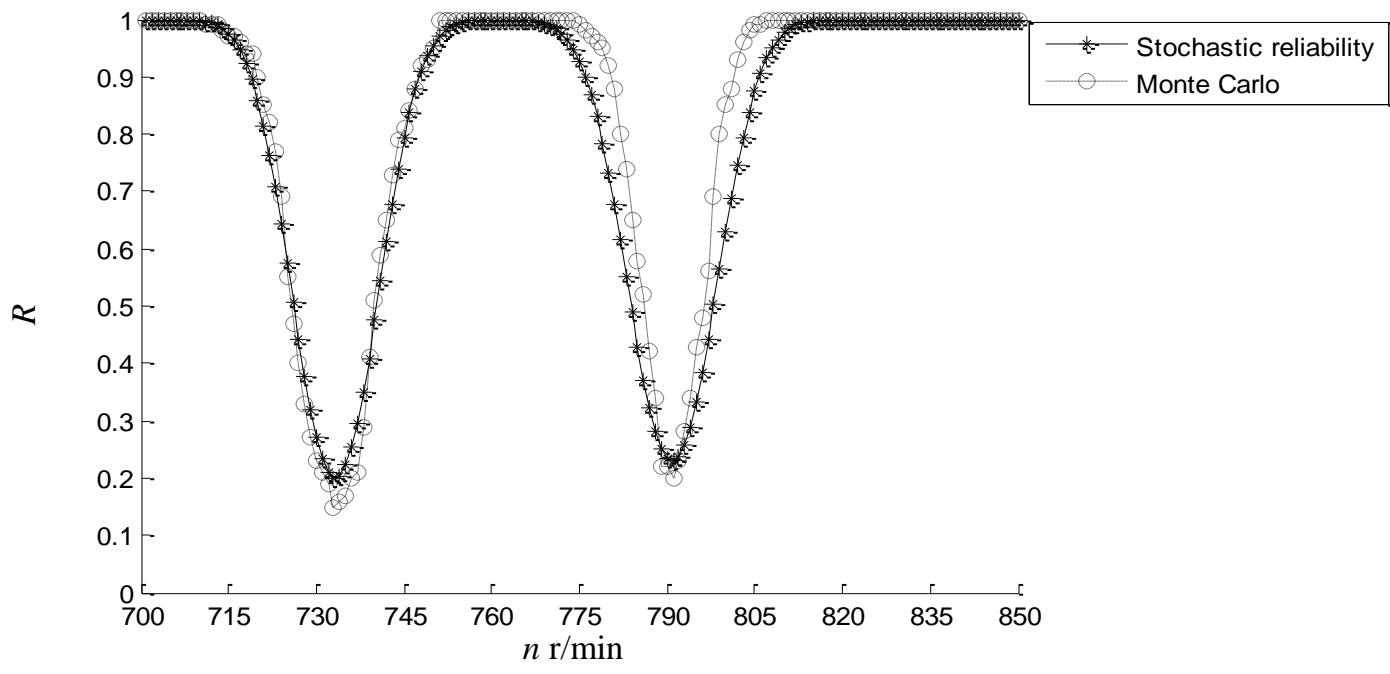

Fig. 3. The contrast figure of the Stochastic reliability and Monte-Carlo

Seeing from the Fig. 3, the results obtained by Monte-Carlo method and Stochastic reliability method are very similar, so the stochastic reliability model is correct and reliable.

\section{Conclusions}

A reliability model of the drum system of a hang type centrifuge under forced vibration failure mode is built by stochastic reliability theory, which reflects the degree of security of the drum system. The lower reliability zones of the drum system of hang type centrifuge are obtained according to 
simulation analysis, where the resonance failure is easy to occur. So these zones should be avoided during the operation of the hang type centrifuge.

\section{Acknowledgement}

This research was financially supported by Guangxi Key Laboratory of Manufacturing System \& Advanced Manufacturing Technology (Grant No. 13-051-09S08) and Guangxi Higher Education Science and Technology Project (YB2014011).

\section{References}

[1] Qi-cai Sun, Centrifuge principle structure and design calculation, Machinery Industry Press: Beijing, 1987. (in Chinese)

[2] Xinwen Tang, Haihui Chen, Investigate on burst accident of rotating drum in centrifuge, Machine Development, 34(3), pp. 104-105, 2005. (in Chinese)

[3] Hua Lu, The research of reliability of ultracentrifuge, Hunan University, 2013. (in Chinese)

[4] Qiwang Zhou, Shuliang Zou, Ping-hui Guo, (Eds). Fault Tree Reliability Analysis of Reprocessing Centrifug, Machinery Design \& Manufacture, (8), pp. 200-206, 2013. (in Chinese)

[5] Yundan Ren, Fuzzy reliability design of high-speed vertical tumbler centrifuge spindle, Journal of Suzhou Vocational University, 17(3), pp. 95-96, 2006. (in Chinese)

[6] Guangheng $\mathrm{Xu}$, Dynamic characteristic research of the spindle system of the hang type centrifuge, Guangxi University, 2015. (in Chinese)

[7] Yigui Ou, Zhilin Li, Shihuang, Hong, An application of the computer simulation to the Mathematical Modelling, Natural Science Journal of Hainan University, 25(1), pp. 1-2, 2004. (in Chinese)

[8] Xianshan Li, Application of Monte Carlo method in reliability analysis, Journal of Astronautics, (2), pp. 69-70, 1983. (in Chinese) 